\title{
Atrial Fibrillation Detection Using MEMS Accelerometer Based Bedsensor
}

\author{
Tero Koivisto $^{1}$, Olli Lahdenoja ${ }^{1}$, Juho Koskinen ${ }^{1}$, Tuukka Panula ${ }^{1}$, Tero Hurnanen ${ }^{1}$, Matti Kaisti ${ }^{1}$, \\ Jere Kinnunen $^{3}$, Pekka Kostiainen ${ }^{3}$, Ulf Meriheina ${ }^{3}$, Tuija Vasankari ${ }^{2}$, Samuli Jaakkola ${ }^{2}$, Tuomas \\ Kiviniemi $^{2}$, Juhani Airaksinen ${ }^{2}$, Mikko Pänkäälä ${ }^{1}$ \\ ${ }^{1}$ Department of Future Technologies, University of Turku, Finland \\ ${ }^{2}$ Heart Center, Turku University Hospital, Finland \\ ${ }^{3}$ Murata Finland Oy, Finland
}

\begin{abstract}
Atrial fibrillation (AFib) is the most common cardiac arrhythmia, affecting eventually up to a quarter of the population. The purpose of this small scale clinical study was to validate the usability of MEMS accelerometer based bedsensor for detection of AFib. A Murata accelerometer based ballistocardiogram bedsensor was attached under the hospital bed magnetically and measurement data was recorded from 20 AFib patients and 15 healthy volunteers, mainly females. The recording time was up 30 minutes. The sensor built-in algorithms automatically extracted features such as heart rate (HR), heart rate variability (HRV), relative stroke volume (SVOL), signal strength (SS) and whether the patient is in bed or not. We calculated median values for each feature HR, HRV, SVOL and SS, and investigated whether it is possible to separate AFib from healthy with these features or their combinations. Areas under the curve (AUC) were 0.98 for full length signals and 0.85 for 3 min signal segments using random forest (RF) classifier corresponding to sensitivity and specificity of $100 \%$ and 93.3\% for full length signals and $90 \%$ and $80 \%$ for $3 \mathrm{~min}$ signals. We conclude, that based on our pilot results, the Murata bedsensor is able to detect AFib, and seems to be a promising technology for long-term monitoring of AFib at home settings as it requires only one-time installation and operational time can be up to years and even tens of years.
\end{abstract}

\section{Introduction}

The development of AFib and its permanent form as well as the prevention of AFib-related strokes are major challenges in cardiology today. AFib is often silent or asymptomatic, but the risk of ischaemic stroke seems to be similar regardless of the presence or absence of symptoms [1]. The bedsensor requires only one-time installation, requires no active user intervention or any attachments to the body of the user and it is capable of long-term at home set- tings. The capability of the bedsensor to detect AFib has been investigated previously with promising results [11] [12] [13] [14]. Also, the research work done in the related field e.g. seismocardiogram and gyrocardiogram to detect AFib using separate device or built-in sensors of the smartphone support the idea that bedsensor could work in AF detection at home setting [15] [16] [17]. Previous research works have been using EmFit-sensor [18] mattress as bedsensor to separate AFib from SR. In this brief, we present our preliminary results using accelerometer-based bedsensor to separate AFib from SR.

\section{Background and motivation}

Asymptomatic AFib patients are more likely to evade diagnostic effort and without appropriate anticoagulation they are left vulnerable to thromboembolism and ischaemic stroke. Approximately one third of all ischaemic strokes are of an unknown cause [1]. Recent studies have shown that more diligent monitoring of heart rhythm with ambulatory devices after a cryptogenic stroke uncovers a high number of silent AFib episodes [4]. The detection of silent AFib is challenging and, therefore, a stroke is still too often the first clinical manifestation of AFib accounting for 22\% of AFib-related strokes [3]. In order to detect symptoms appearing at periodic or random intervals, a capability for longer-term monitoring, e.g. for several days or weeks at a time, is required. Implantable ECG loop recorders perform well in AFib detection, but they are hardly feasible in large patient cohorts due to invasive nature and costs. Thus, there is an unmet clinical need for better AFib detection tools. Today, the AF detection tools could be classified in three groups:

- Intermittent monitoring. This type of techniques or devices rely on spot measurents e.g. patient takes selfrecordings himself/herself ideally on a daily basis. The simplest approach is probably the self-palpation for example from the wrist [4]. Different technological solutions are also available such as intermittent ECG recording using 
smartphone and simple add-on using recording from the fingers of the person, smartphone camera based solutions wherein the patients puts finger to the smartphone [6] [7] and smartphone built-in MEMS motion sensor based solution wherein the patient takes the mesurement from the chest area [4][19].

- Wearable monitoring. Normal ECG recording or 24hholter monitoring are current gold standard methods for AFib detection. Some Holter monitors also offer capability for monitoring ECG up to 2 weeks [9]. Recently, the smart watches such as AppleWatch measuring PPG from the wrist have been proposed [10]. The AppleWatch also includes option to measure single-lead ECG from the crown of the watch. In this case, the idea is that watch continuously monitors heart rate from the wrist using PPG and if heart rate seems to be irregular, the alert is given to the user, and the user can take spot ECG from the watch to validate the alert. In principle, watch can be used for a long time but it needs active user intervention as the device must be charged regularly. Also, the watch should be fastened tighly to the wrist to get high quality signal from the PPG.

- Implantable devices. Implantable ECG loop recorders perform very well in AFib detection and are operational up to three years [8], but they are hardly feasible in large patient cohorts due to invasive nature and costs.

To conclude, current AFib detection either require attachment of electrodes to patients (skin irritation, limited operational time), are not practical for screening masses (implantable device), are not comfortable in long-term use (smartwatch, Holter) or require active user intervention (spot measurements, charging smartwatch) making these detection tools unoptimal for long-term cardiac monitoring at home. This challenge could be addressed by developing unobtrusive cardiac monitoring techniques based on ballistocardiogram based bedsensors.

\section{Methods}

We used Murata BCG sensor (SCA10/11H) shown in Figure 2. which is able to measure small movement of the whole body caused by the person's beating heart e.g. $\mathrm{BCG}$ signal. In order to measure the BCG signal the sensor has an accelerometer having ultra low-noise level of 15 $\mu \mathrm{g} / \sqrt{\mathrm{Hz}}$. The sensor has two modes: raw data and result mode. In raw data mode, the raw data is collected without any signal and data processing. In the result mode, the sensor directly outputs heart rate (HR), heart rate variability (HRV), relative stroke volume (SVOL), respiration (RR), signal strength (SS) and whether the patient is in bed or not. The bedsensor was attached under the hospital bed magnetically and measurement data was recorded from 20 AFib patients and 15 healthy volunteers, mainly females. The AFib patients had permanent AFib and most

\begin{tabular}{|c|c|c|c|c|}
\hline \multicolumn{5}{|c|}{ HEALTHY PATIENTS } \\
\hline PATIENT ID & HR (bpm) & RR (1/min) & SVOL & HRV \\
\hline 1 & 64 & 14 & 36 & 71 \\
\hline 2 & 55 & 16 & 62 & 19.5 \\
\hline 3 & 58 & 15 & 44 & 41 \\
\hline 4 & 67 & 14 & 40 & 67 \\
\hline 5 & 67 & 12 & 64 & 52.5 \\
\hline 6 & 70 & 20 & 41 & 44 \\
\hline 7 & 59 & 16 & 48 & 68 \\
\hline 8 & 60 & 11 & 42 & 28.5 \\
\hline 9 & 55 & 10 & 62 & 27 \\
\hline 10 & 76 & 14 & 46 & 81 \\
\hline 11 & 74 & 15 & 64 & 60 \\
\hline 12 & 51 & 10 & 53 & 53 \\
\hline 13 & 78 & 14 & 33 & 73 \\
\hline 14 & 54 & 12 & 64 & 35 \\
\hline 15 & 50 & 15 & 91 & 27 \\
\hline \multicolumn{5}{|c|}{} \\
\hline
\end{tabular}

Table 1. Sensor output for healthy patients.

of them had heart failure, coronary artery disease or both. The recording time was up 30 minutes and data was collected in raw data mode. We investigated both raw and result mode data; the raw data was fed to the Murata algorithm and the algorithm outputted automatically HR, HRV, SVOL, RR, SS and whether the patient is in bed or not.

\section{4. $\quad$ Results}

We calculated median values for each feature HR, HRV, SVOL and SS, and investigated whether it is possible to separate AFib from healthy with these features or their combinations for full 30 and 3 minute signals in result mode of the sensor. Tables 1 and 2 show the most important calculated median values for healthy and atrial fibrillation patients. Areas under the curve (AUC) were 0.63 for HR, 0.92 for HRV, 0.76 for SVOL and 0.70 for SS for full length signals. AUC for all features combined e.g. HR, HRV, SVOL and SS was 0.98 for full length signals using RF classifier corresponding to sensitivity and specificity of $100 \%$ and $93.3 \%$. Similarly, the AUCs for cropped 3 min caption of signals were 0.58 for $\mathrm{HR}, 0.82$ for HRV, 0.67 for SVOL and 0.66 for SS. AUC for all features combined with the 3 minute signals was 0.85 with sensitivity and specificity of $90 \%$ and $80 \%$. The corresponding ROC curves are shown in Figures 3 and 4.

We tested also our custom built algorithm for AFib detection without using the parameters calculated automatically from the bedsensor [19]. Figure 1 shows preprocessed raw data from sinus rhythm (SR) and atrial fibrillation (AFib), and the resulting $5 \mathrm{~s}$ autocorrelation result in both cases. In raw data mode the BCG data were first preprocessed by applying a band-pass filter to remove signal noise and bias. The algorithm then examined the signal 
Sinus Rhythm
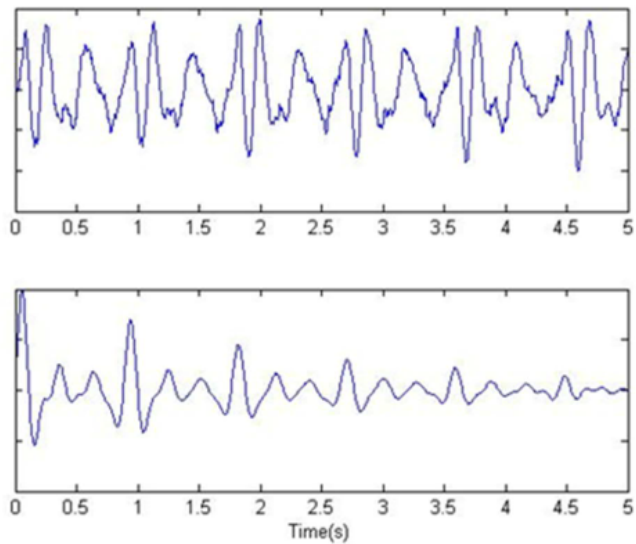

Atrial Fibrillation
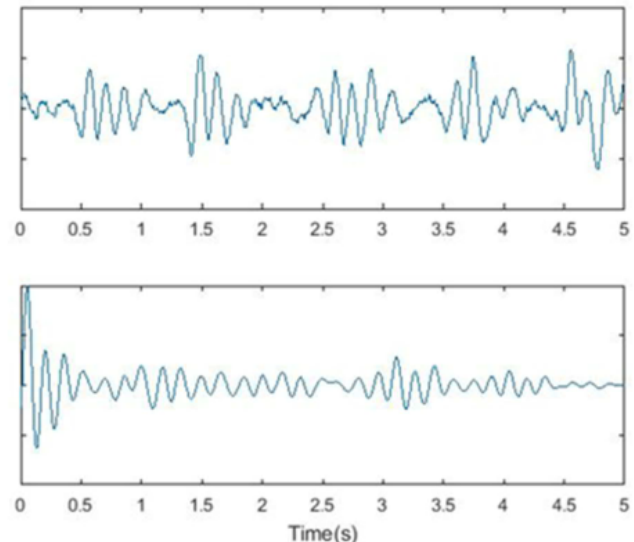

Figure 1. Sinus rhythm and atrial fibrillation BCG signals with 5 s autocorrelation results.
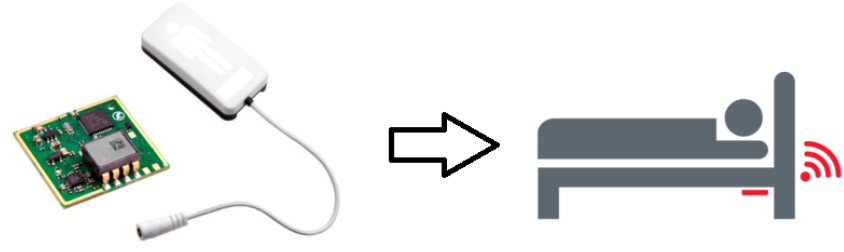

Figure 2. Murata BCG sensor SCA10/11H.

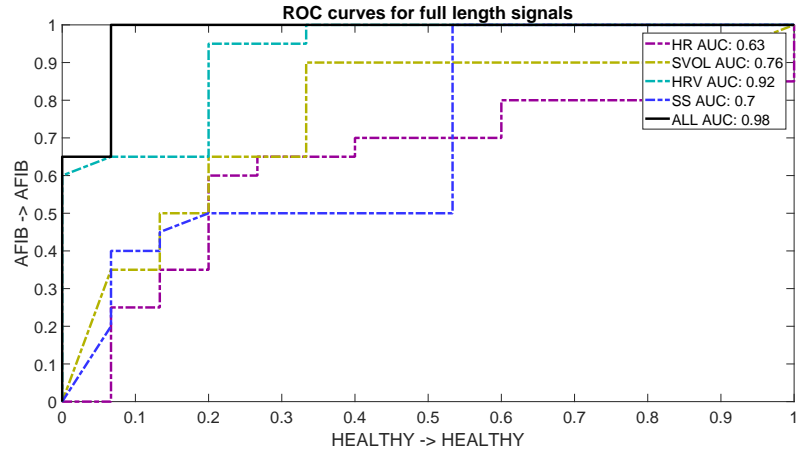

Figure 3. ROC curve for full length signals with random forest classifier.

with $5 \mathrm{~s}$ autocorrelation windows to find evidence of constant beat-to-beat intervals. In order to classify the signal as AFib or SR, the share of signal segments with regularity was determined. If the share of the segment with regularity was over $10 \%$, the signal was classified as SR, otherwise it was AFib. The resulting sensitivity and spesificity was 95\% and 100\% for both full 30 and 3 minute signal segments.

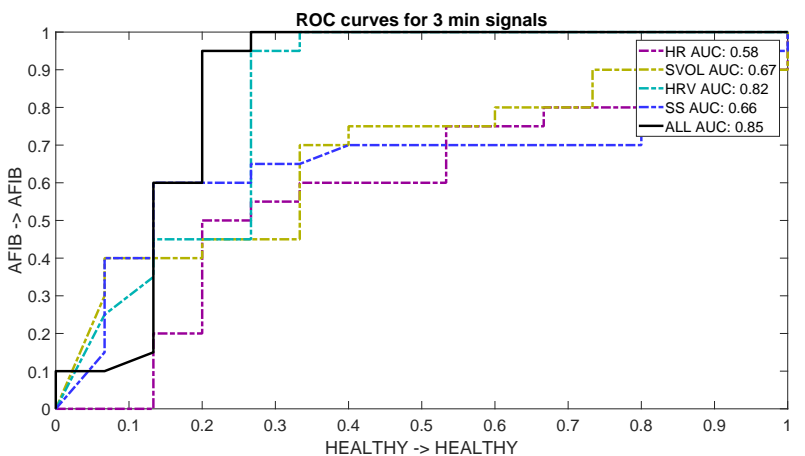

Figure 4. ROC curve for 3 minutes length portion of the signals with random forest classifier.

\section{Discussion}

In addition to RF classifier, kernel support vector machine (KSVM) gave similar but slightly weaker results based on the data. As the sample size in this study was quite small and the control group used consisted of healthy individuals, also persons with cardiac disease should also be included to the control group in future studies. Also, the subjects in our case were adviced to remain still during the measurement. In a more general case the algorithm should be able to automatically both detect if the person is in bed and whether the person is still to enable good quality output from the AFib detection. Subsequently, future work includes developing efficient means for real-time motion artefact removal and subsequent processing of the data. 


\begin{tabular}{|c|c|c|c|c|}
\hline \multicolumn{5}{|c|}{ AFIB PATENTS } \\
\hline PATIENT ID & HR (bpm) & RR (1/min) & SVOL & HRV \\
\hline 1 & 73 & 13 & 76 & 107 \\
\hline 2 & 87 & 13 & 85 & 80 \\
\hline 3 & 81 & 17 & 82 & 82 \\
\hline 4 & 87 & 15 & 81 & 70 \\
\hline 5 & 85 & 10 & 115 & 72 \\
\hline 6 & 86 & 17 & 57 & 75 \\
\hline 7 & 54 & 8 & 75 & 128 \\
\hline 8 & 63 & 12 & 74 & 131 \\
\hline 9 & 69 & 12 & 39 & 114 \\
\hline 10 & 68 & 15 & 50 & 99 \\
\hline 11 & 71 & 15 & 36 & 94 \\
\hline 12 & 72 & 13 & 79 & 101 \\
\hline 13 & 66 & 15 & 63 & 84 \\
\hline 14 & 69 & 12 & 56 & 124 \\
\hline 15 & 70 & 10 & 54 & 120 \\
\hline 16 & 73 & 16 & 35 & 91 \\
\hline 17 & 60 & 12 & 70 & 111 \\
\hline 18 & 71 & 13 & 78 & 80 \\
\hline 19 & 78 & 14 & 51 & 92 \\
\hline 20 & 65 & 11 & 58 & 111 \\
\hline & & & & \\
\hline
\end{tabular}

Table 2. Sensor output for atrial fibrillation patients.

\section{Conclusion}

Based on our pilot results, the Murata bedsensor is able to separate AFib from SR also in result mode, and seems to be a promising technology for long-term monitoring of AFib at home settings as it requires only one-time installation and operational time can be up to years or even tens of years.

\section{References}

[1] Kirchhof P et.al., 2016 ESC Guidelines for the Management of Atrial Fibrillation Developed in Collaboration with EACTS. Eur Heart J. 2016.

[2] Sanna T, Diener HC, Passman RS, Di Lazzaro V, Bernstein RA, Morillo CA, Rymer MM, Thijs V, Rogers T, Beckers F, Lindborg K, Brachmann J; CRYSTAL AF Investigators. Cryptogenic Stroke and Underlying Atrial Fibrillation. N Engl J Med. 2014 Jun

[3] Jaakkola J, Mustonen P, Kiviniemi T, Hartikainen JE, Palomäki A, Hartikainen P, Nuotio I, Ylitalo A, Airaksinen KE. Stroke as the First Manifestation of Atrial Fibrillation. PLoS One. 2016

[4] https://www.tunnepulssisi.fi/

[5] https://cardiosignal.com/

[6] https://www.preventicus.com/

[7] https://www.fibricheck.com/

[8] https://www.medtronic.com/us-en/patients/treatmentstherapies/heart-monitors/our-monitors/reveal-linqicm.html

[9] https://www.irhythmtech.com/

[10] https://www.apple.com/apple-watch-series-5/health/
[11] C. Bruser et al., A Feasibility Study on the Automatic Detection of Atrial Fibrillation using an Unobtrusive BedMounted Sensor, in Comput. Cardiol. Conf. (CinC), 2011.

[12] C. Bruser et al., Automatic Detection of Atrial Fibrillation in Cardiac Vibration Signals, IEEE J. Biomed. Health Inform., vol. 17, no. 1, pp. 162-171, 2013.

[13] M. D. Zink et al., Heartbeat Cycle Length Detection by a Ballistocardiographic Sensor in Atrial Fibrillation and Sinus Rhythm, BioMed Res. Int., vol. 2015.

[14] Xin Wen, Yanqi Huang, Xiaomei Wu, and Biyong Zhang, A Feasible Feature Extraction Method for Atrial Fibrillation Detection from BCG, IEEE Journal of Biomedical and Health Informatics 2019.

[15] Hurnanen T, Lehtonen E, Jafari Tadi M, Kuusela T, Kiviniemi T, Saraste A, Vasankari T, Airaksinen J, Koivisto T, Pankaala M. Automated Detection of Atrial Fibrillation Based on Time-Frequency Analysis of Seismocardiagrams. IEEE J Biomed Health Inform. 2016 Nov.

[16] Lahdenoja O, Hurnanen T, Iftikhar Z, Nieminen S, Knuutila T, Saraste A, Kiviniemi T, Vasankari T, Airaksinen J, Pankaala M, Koivisto T. Atrial Fibrillation Detection via Accelerometer and Gyroscope of a Smartphone. IEEE J Biomed Health Inform., 2017.

[17] Pankaala M, Koivisto T, Lahdenoja O, Kiviniemi T, Saraste A, Vasankari T, Airaksinen J. Detection of Atrial Fibrillation with Seismocardiography. Conf Proc IEEE Eng Med Biol Soc. 2016.

[18] https://www.emfit.com/

[19] Jaakkola, J., Jaakkola, S., Lahdenoja, O., Hurnanen, T., Koivisto, T., Pänkäälä, M., Knuutila, T., Kiviniemi, T., Vasankari, T., Airaksinen, K. E. J. Mobile Phone Detection of Atrial Fibrillation with Mechanocardiography: The Mode-AF study (Mobile Phone Detection of Atrial Fibrillation). Circulation, 137(14), 1524-1527, 2018.

\section{Acknowledgements}

This study was funded by Business Finland's Medical Mems Valley -project (543/31/2015).

Address for correspondence:

Tero Koivisto

Department of Future Technologies, University of Turku tejuko@utu.fi 